\title{
A 50-year-old male with fever, cough, dyspnoea, chest pain, weight loss and night sweats
}

\section{Case report}

A 50-year-old, male patient presented during the day for an urgent consultation. For over 10 weeks, he had had a history of high-grade fever $\left(39^{\circ} \mathrm{C}\right)$, cough with mucopurulent expectoration, shortness of breath, right-sided chest pain, weight loss (>10\%) and night sweats.

General examination showed a thin body build, pale skin and extreme dyspnoea with oxygen saturation of $77 \%$. An examination of the chest revealed air and fluid in the pleural space on the right side of the body. All other systems were checked and were all clinically normal.

A posteroanterior chest radiography was performed and showed an air-fluid level in the right hemithorax and a minimal shift of mediastinum towards the left (figure 1).

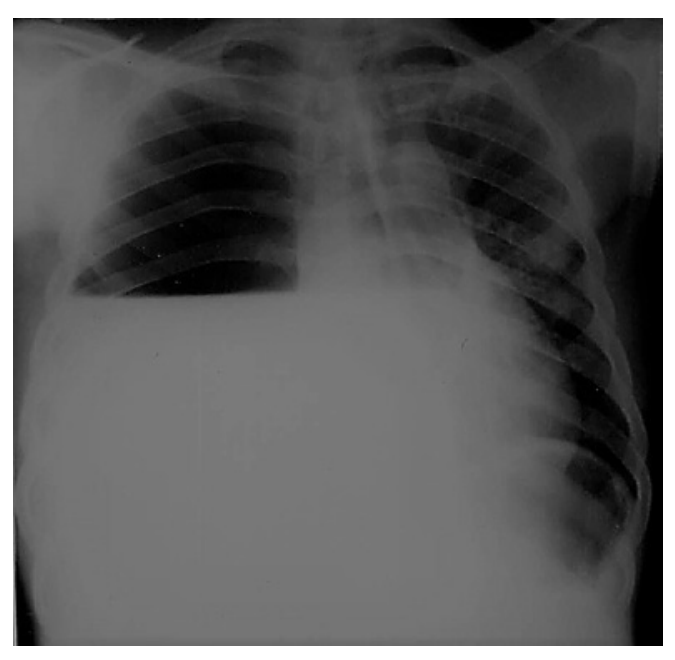

Cite as: Muntean PE. A 50-year-old male with fever, cough, dyspnoea, chest pain, weight loss and night sweats. Breathe 2017; 13: 43-48.
Figure 1 Chest radiograph.

\section{Task 1}

Based on this information, what is the likely diagnosis?
a. Empyema
b. Chylothorax
c. Hydropneumothorax
d. Pseudochylothorax 


\section{Answer 1}

c. Hydropneumothorax

"Hydropneumothorax" is the term given to the concurrent presence of a pneumothorax and a hydrothorax (air and fluid) in the pleural space. Hydrothorax is the presence of noninflammatory serous fluid within the pleural cavity. The fluid in the pleural cavity causes compression of lung with resulting dyspnoea and ventral absence of lung sounds. Pneumothorax refers to the presence of air in the pleural space. When this collection of air constantly enlarges with resulting compression of mediastinal structures, it can be life-threatening and is known as a tension pneumothorax [1].

Task 2

What can cause hydropneumothorax?
a. Thoracic trauma
b. Bronchopleural fistula
c. Oesophagopleural fistula
d. Mycobacterium tuberculosis
e. All of the above 


\section{Answer 2}

e. All of the above

A sputum sample was collected and examined by Neelsen staining. Based on the radiography findings, a computed tomography body scan and a chest ultrasound were requested to guide pleural puncture (figure 2).

Routine blood and urine tests and a thoracentesis were performed. Approximately $1050 \mathrm{~mL}$ of pleural liquid was obtained and samples were sent to the laboratory, for the culture of pleural pus on Lowenstein Jensen medium and incubated for a period of 2 months [2].
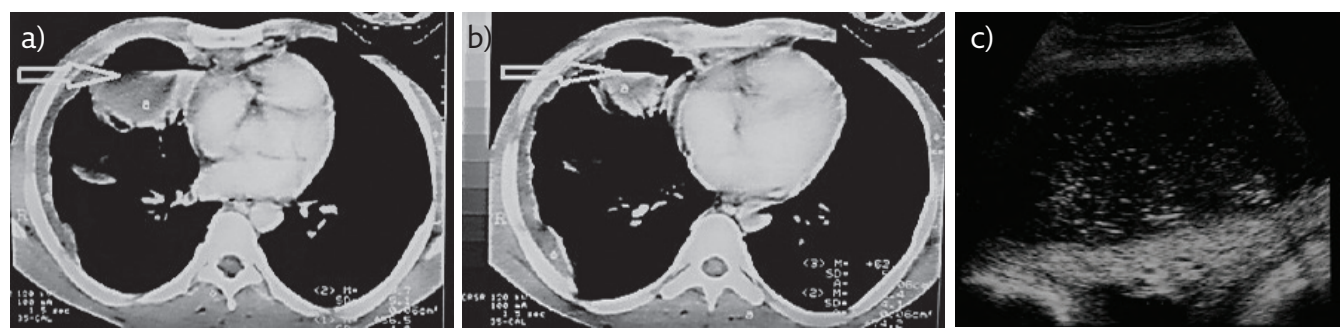

Figure 2 a and b) Computed tomography. c) Chest ultrasound.

\section{Task 3}

What can be diagnosed by correlating the chest radiography, thoracic echography and computed tomography body scan results?

a. Pleural and pericardial effusion.

b. Parapneumonic pleural effusion.

c. Large, right-side pleural effusion with displacement of the mediastinum as well as thickening of the pleura and an underlying disease process; fine internal echoes in the pleural collection, which strongly suggests infected fluid. 


\section{Answer 3}

c. Large, right-side pleural effusion with displacement of the mediastinum as well as thickening of the pleura and an underlying disease process; fine internal echoes in the pleural collection, which strongly suggests infected fluid.

Routine blood tests revealed $91 \%$ lymphocytes, $8.9 \mathrm{~g} \cdot \mathrm{dL}^{-1}$ haemoglobin, and 10000 leukocytes per $\mathrm{mm}^{3}$ with $78 \%$ neutrophils and $7 \%$ eosinophils. Urine test results were normal. There was a 17-mm positive Mantoux reaction. Ziehl Neelsen staining of sputum showed acid-fast bacilli (figure 3) and culture of pleural pus on Lowenstein Jensen medium showed rough, dry and raised colonies with a wrinkled surface.

Microscopy and culture of pleural fluid did not show any other fungus or bacteria.

Pyopneumothorax is an uncommon condition with a combination of air and pus within the pleural space. Frequently, emphysema may precede a pneumothorax but certain underlying conditions, like tuberculosis, may increase the risk of chronic lung and some pleural diseases. Usually, tuberculosis with pyopneumothorax determine complications due to the rupture of a cavity into the pleural space so these complications make the treatment management even more difficult. The tuberculosis treatment must be given as national protocols require.

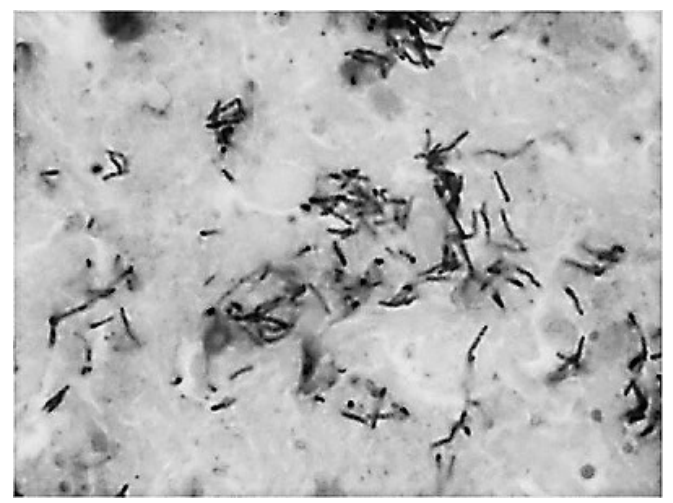

Figure 3 Sputum sample. Ziehl Neelsen staining.

\section{Task 4}

Typically, the treatment for pyopneumothorax is:

a. Therapeutic thoracentesis with wide drainage and general antibiotics for 2 months.

b. Referral to a thoracic surgeon for ligation of the thoracic duct.

c. Thoracoscopy with pleural drainage and pleural biopsy. 


\section{Answer 4}

a. Therapeutic thoracentesis with wide drainage and general antibiotics for 2 months.
Typically, the treatment for pyo-pneumothorax is therapeutic thoracentesis with wide drainage and general antibiotics suitable for the identified organisms. This treatment needs to be given for almost 2 months [3]. If these methods of treatments fail, surgical evaluation may be required.

\section{Task 5}

In this case, which tuberculosis treatment scheme applies?

a. 2 HRZE or 2 HRZS $7 / 7+4$ HR 3/7

b. 2 HRZSE + 1 HRZE $7 / 7+5$ HRE $3 / 7$

c. $2 \mathrm{HRZ} 7 / 7+4 \mathrm{HR} 3 / 7$

$\mathrm{H}$ : isoniazid; R: rifampicin; Z: pyrazinamide; E: ethambutol; S: streptomycin. 


\section{Answer 5}

a. 2 HRZE $7 / 7+4$ HR $3 / 7$

Isoniazid, rifampicin, ethambutol and pyrazinamide daily for 2 months (2 HRZE 7/7) followed by 4 months of isoniazid and rifampicin given three times a week (4 HR 3/7) is appropriate to treat pulmonary tuberculosis in a new case using treatment regimen 1. Drug doses were adapted to the weight of the patient according to national guidelines.

Based on the clinical and paraclinical findings, this was confirmed to be a rare case of pyopneumothorax in an active tuberculosis patient. There was good clinical response to antituberculous therapy.

\section{Conflict of interest}

None declared.

\section{References}

1. Light RW. Pleural Effusion. www.merckmanuals.com/ professional/pulmonary-disorders/mediastinal-and-pleuraldisorders/pleural-effusion Date last updated: October 2017

2. National Institute of Pneumology "MARIUS NASTA". The Program for Prevention, Supervision and Control of

Tuberculosis, 2015 Methodological Guide. Bucharest, Ministry of Health, 2015.

3. Duzhyi ID. Treatment of acute pyo-pneumothorax during the tuberculosis epidemic. Klinkhir 2003; 9: 34-37. 